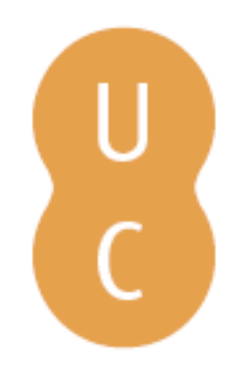

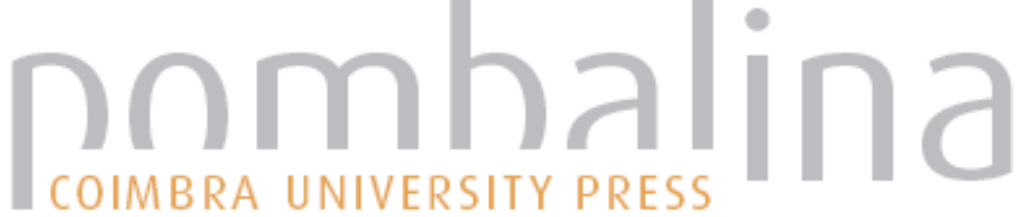

\section{Promoción de la lectura y politicas de alfabetización informacional}

Autor(es): $\quad$ Soria, Maria Gladys Ceretta

Publicado por: Imprensa da Universidade de Coimbra

URL

persistente: URI:http://hdl.handle.net/10316.2/31918

DOI: $\quad$ DOI:http://dx.doi.org/10.14195/978-989-26-0869-3_19

Accessed : $\quad$ 26-Apr-2023 14:05:52

A navegação consulta e descarregamento dos títulos inseridos nas Bibliotecas Digitais UC Digitalis, UC Pombalina e UC Impactum, pressupõem a aceitação plena e sem reservas dos Termos e Condições de Uso destas Bibliotecas Digitais, disponíveis em https://digitalis.uc.pt/pt-pt/termos.

Conforme exposto nos referidos Termos e Condições de Uso, o descarregamento de títulos de acesso restrito requer uma licença válida de autorização devendo o utilizador aceder ao(s) documento(s) a partir de um endereço de IP da instituição detentora da supramencionada licença.

Ao utilizador é apenas permitido o descarregamento para uso pessoal, pelo que o emprego do(s) título(s) descarregado(s) para outro fim, designadamente comercial, carece de autorização do respetivo autor ou editor da obra.

Na medida em que todas as obras da UC Digitalis se encontram protegidas pelo Código do Direito de Autor e Direitos Conexos e demais legislação aplicável, toda a cópia, parcial ou total, deste documento, nos casos em que é legalmente admitida, deverá conter ou fazer-se acompanhar por este aviso. 
Maria Manuel Borges

Elias Sanz Casado

Coordenação

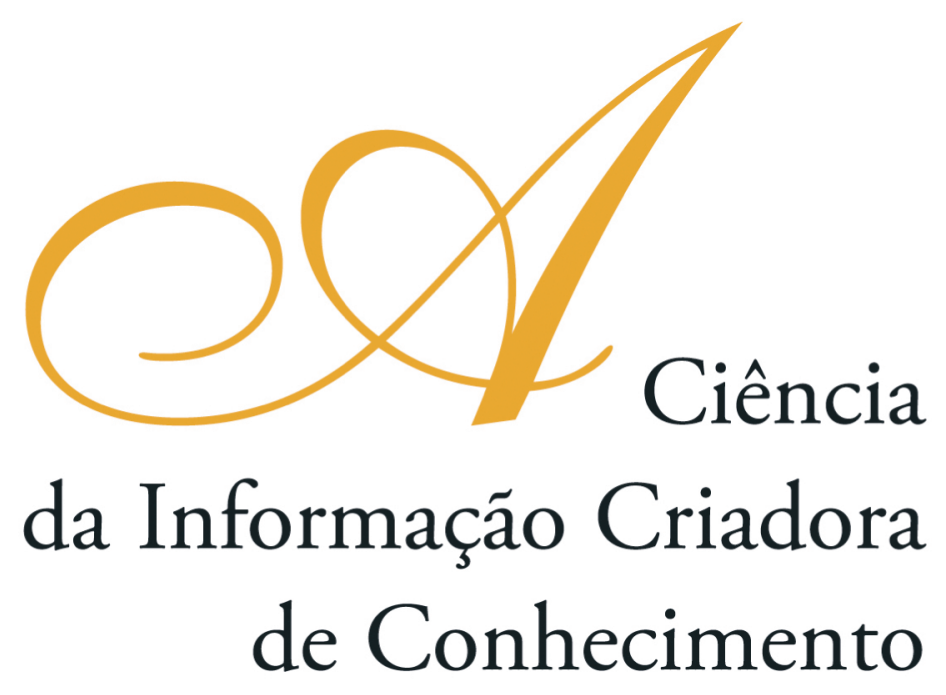

Vol. I I

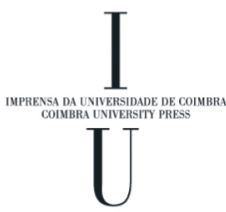

- COImbra 2009 


\title{
Promoción de la lectura y politicas de Alfabetización InformaCional
}

\author{
Maria Gladys Ceretta Soria \\ Universidad de la República (Uruguai)
}

\section{Resumen}

La presente ponencia apunta a reflexionar sobre la relación entre la promoción de la lectura y la alfabetización informacional, enfocadas como política pública en el marco de las acciones emprendidas por el Plan Nacional de Lectura de Uruguay, con la finalidad de contribuir a la equidad social y a la democratización de la información y el conocimiento. La temática es objeto de estudio de una línea de investigación que se viene desarrollando en la Escuela Universitaria de Bibliotecologia y Ciencias Afines de la Universidad de la República. Los contextos propios de la actual "Sociedad de la Información y el Conocimiento" se vuelven determinantes a la hora de formar individuos que puedan integrarse plenamente a lo largo de toda su vida, a los desafíos que ésta presenta. La lectura es una de las herramientas fundamentales que le permiten al individuo ser parte de la sociedad y participar activamente de los cambios que en ella se producen. Los nuevos contextos sociales fuertemente pautados por la presencia de las tecnologías de la información y la comunicación requieren de múltiples alfabetizaciones. Estar alfabetizado implica no sólo saber leer y escribir, sino tener habilidades para desarrollar competencias que permitan comprender el mundo exterior. El manejo de la información con sentido crítico se vuelve clave para formar individuos autónomos, capaces de interpretar, crear y evaluar el mundo que los rodea, aptos para enfrentar los nuevos desafíos que presenta ésta sociedad. La Alfabetización Informacional adquiere dimensiones insospechadas para formar actores sociales calificados. Las políticas públicas de promoción de la lectura a nivel de los distintos países, generalmente se enfocan hacia una de las dimensiones de la lectura, la recreativa. Sin embargo, cada vez más es necesario que estas políticas se aborden desde una perspectiva global que involucre la alfabetización en el uso de la información como un elemento primordial para garantizar la formación de un individuo libre, autónomo y crítico. Estos son algunos de los fundamentos que permiten afirmar que los Planes de Lectura deberían contemplar políticas de Alfabetización Informacional. Ellas permitirían contribuir a la formación de usuarios-lectores en el sentido más amplio de los términos.. La investigación propuesta potencia, redimensiona y revaloriza la importancia de la biblioteca como ámbito natural de promoción de la lectura y de implementación de políticas de Alfabetización Informacional y la presencia fundamental de los profesionales Bibliotecólogos, en este contexto, desde una mirada interdisciplinar. Se pretende enfocar el tema de la promoción de la lectura en el marco de la Alfabetización Informacional, considerando que pueden ser múltiples las actividades que realice el Bibliotecólogo para formar al usuario al mismo tiempo que a través de esa formación está contribuyendo a las políticas y planes de promoción de la lectura. Se realiza una breve revisión de los conceptos teóricos de Sociedad de la información y el Conocimiento, Alfabetización Informacional, Lectura y Promoción de la Lectura y se analizan los antecedentes y evolución de las acciones a favor de la la lectura en Uruguay con especial énfasis en el Plan Nacional de Lectura. Se concluye que es posible vincular las políticas de promoción de la lectura con las de Alfabetización Informacional y que la propuesta podría favorecer ampliamente la articulación y coordinación con las políticas informativas, educativas y sociales a nivel nacional. 


\section{Abstract}

The objective of this article is to reflect upon the relationship between reading promotion and information literacy, regarded as a public policy in the context of the National Reading Plan in Uruguay. The objective of this Plan is to contribute to social equity and to information and knowledge democratization access.

This subject is being object of research at Escuela Unviersitaria de Bibliotecología y Ciencias Afines in Universidad de la República where this paper summarizes the theoretical approach which founds this research line. The Information and Knowledge Society requires of individuals able to integrate and adapt themselves all along their lives. Reading is regarded as a fundamental tool which allows people to integrate and to participate actively into the society and to become a promoter of its changes. The new social organization featured by the presence of information and communication technologies requires of several "literacies". Nowadays, being literate implies not only being able to write and read but also having competences in order to understand what is happening in the outer world. Being able to use information with critical sense is a key issue in order to promote autonomous individuals, able to think, to analyze, to create and evaluate everything around themselves, as well as to face the new society challenges. In this context, the information literacy acquires relevance in order to promote the education of qualified social actors.

It is noted that national public policies are orientated to leisure aspects of reading. However, it is pointed out that these policies should be regarded since a global perspective tending to include the literacy in the use of information in order to promote an open minded individual. All of these aspects are the foundations which allow to state that Reading Plans should contemplate Information Literacy Policies. These policies would contribute to achieve a society with usersreaders in an ample sense. This research enhances and puts emphasis on the library, as the natural ambit of reading promotion, and as the space where informational literacy policies should be implemented. In this sense, the presence of librarians who can contribute with their experience and knowledge is fundamental.

The intention is to focus reading in the context of information literacy, taking into account that librarians can carry out multiple activities in order to contribute to this objective.

A literature review was carried out in order to present some important concepts about Information and Knowledge Society, Information Literacy, Reading and Reading Promotion. The case of Uruguay is presented and the evolution of the actions is analyzed. It is concluded in the possible relationship between reading promotion and information literacy policies. This perspective is recommended in order to articulate and coordinate different policies as the educative, social ones on a national scenario.

\section{Introducción}

\subsection{Antecedentes}

Desde el año 2005 la Escuela Universitaria de Bibliotecología y Ciencias Afines (EUBCA) de la Universidad de la República, viene participando activamente de las acciones que lleva adelante el Plan Nacional de Lectura impulsado por la Dirección de Educación del Ministerio de Educación y Cultura. Por resolución de ese Ministerio la EUBCA, junto con otras instituciones públicas y privadas, así como diversos actores sociales vinculados a la lectura, integra la Comisión Asesora del Plan Nacional de Lectura de Uruguay (PNL). En este contexto, la EUBCA viene implementando diferentes programas de promoción de la lectura con la participación de docentes y 
estudiantes de la Licenciatura en Bibliotecología. La experiencia integra actividades de promoción de la lectura y formación de lectores en habilidades y destrezas en el manejo de información.

Desde la EUBCA se ha manifestado continuamente la necesidad de fortalecer y crear bibliotecas, en especial públicas, populares y escolares, como ámbitos naturales de implementación de las acciones de promoción de la lectura a nivel nacional. En esa línea, se pretende revalorizar el rol de los bibliotecólogos como mediadores calificados para promover la lectura en su más amplia expresión y como profesionales aptos para implementar políticas de Alfabetización Informacional, que se materialicen a través de acciones que conduzcan a la formación de usuarios-lectores hábiles para relacionarse con la información.

Como Profesora Titular de la EUBCA, a cargo del dictado de la asignatura "Servicio de Información y Consulta", en la Licenciatura en Bibliotecología, es nuestra responsabilidad formar a los estudiantes de grado, en las temáticas relacionadas con la selección, uso y evaluación de recursos de información. Asimismo, el programa del mencionado curso introduce al estudiante en el conocimiento de las actuales políticas de Alfabetización Informacional y resignifica el valor de la formación de usuarios de la información como un elemento clave en los actuales contextos sociales. En ese sentido, se promueve la importancia de dar al usuario-lector la capacitación adecuada en el uso, selección y evaluación de la información.

Asimismo, a nivel de la EUBCA se ha generado una línea de investigación sobre promoción de la lectura, de la que participan docentes de diversas áreas temáticas y se ha incorporado además, la presencia estudiantes avanzados de la Licenciatura en Bibliotecología. Este es el contexto en el cual la autora está investigando el tema "Promoción de la Lectura y Alfabetización Informacional" y sobre el cual realiza está realizando su tesis doctoral.

\subsection{Justificación de la propuesta}

El surgimiento de un nuevo tipo de sociedad a la que desde hace algo más de veinte años se le ha denominado "sociedad de la información" y actualmente "sociedad de la información y el conocimiento" está fuertemente pautado por el desarrollo y avance de las tecnologías de la información y la comunicación que han generado un proceso de globalización de la información. Este proceso ha promovido la facilidad de acceso, uso, distribución, almacenamiento y creación de recursos de información disponibles y compartidos a nivel mundial. A pesar de ello, hablar hoy, de una sociedad global de la información es casi un imposible, puesto que cada país construye un universo social propio de acuerdo a su cultura, su historia y sus particularidades. Dice MATTELART:

No puede haber sociedad global de la información. Es un mito. Puede haber sociedades del conocimiento porque cada país se introduce en el universo tecnológico a partir de su cultura, su historia y la especificidad de sus instituciones. (MATTELART, 2004).

Los nuevos escenarios de la sociedad de la información y el conocimiento repercuten directamente sobre la educación formal y en especial potencian la necesidad de una 
educación informal a lo largo de toda la vida del individuo para garantizar la equidad y la inclusión social. ${ }^{1}$

La lectura se vuelve una herramienta fundamental que facilita el acceso del individuo a la información al conocimiento y en general, al mundo que lo rodea. Asimismo, favorece la educación continua a lo largo de toda la vida y garantizando su inclusión social. Las tecnologías de la información y la comunicación han generado la necesidad de apuntar a múltiples alfabetizaciones. Es necesario formar individuos autónomos, capaces de interpretar, crear y evaluar el mundo que los rodea, aptos para enfrentar los desafíos presentes y futuros. En este contexto estar alfabetizado en información es un valor agregado fundamental para ser parte activa de la nueva sociedad.

Las políticas públicas de promoción de la lectura a nivel de los distintos países, generalmente se enfocan hacia una de las dimensiones de la lectura, la recreativa. Sin embargo, se hace cada vez más necesario que las mismas se aborden desde una perspectiva integral que considere a la lectura en el sentido más amplio como una herramienta de acceso a la información y al conocimiento. A través de la Alfabetización Informacional se puede alcanzar este objetivo.

No se debe perder de vista la importancia de las bibliotecas (en especial de la Biblioteca Pública) como espacios de promoción de la lectura y de implementación de políticas de Alfabetización Informacional. Por lo tanto, las mismas deben ser revalorizadas y redimensionadas en cuanto al lugar que ocupan en las estructuras de información de los países, así como su transformación en función de los nuevos entornos digitales. Para lograr estos objetivos se requiere la presencia de los profesionales Bibliotecólogos en un trabajo integrado e interdisciplinar. Al decir de MARZAL (2004) son muchas las áreas del conocimiento que están implicadas en la sociedad de la información, "pero indudablemente entre ellas están con peso específico propio las Ciencias de la Documentación”.

En este sentido, se pretende fundamentar el por qué y para qué, los Planes de Lectura deberían contemplar políticas de Alfabetización Informacional, como parte de las acciones en favor de la promoción de la lectura. La incorporación de políticas de Alfabetización Informacional permitiría enfocar la formación de lectores en el sentido más amplio del tema, y la lectura sería la herramienta para lograrlo. Las bibliotecas deberían ser los espacios naturales de implementación de las acciones de promoción de la lectura y Alfabetización Informacional.

\section{Objetivo}

A través de la presente ponencia se pretende reflexionar sobre la importancia de la promoción de la lectura como política pública en el contexto de la sociedad de

${ }^{1}$ Los sistemas educativos tienen que estar preparados para "formar para lo desconocido" Al decir del ex Rector de la Universidad de la República y ex Ministro de Educación y Cultura del Uruguay, Ing. Jorge Brovetto (1994), la Universidad debe formar individuos profesionales, pensantes, que sean capaces de actuar y enfrentarse en el día a día con lo desconocido, cada día es un aprendizaje para el que hay que estar preparado. 
la información y el conocimiento, y fundamentar el vínculo con la Alfabetización Informacional. Este objetivo se enmarca en la investigación que la autora viene realizando como tesis doctoral cuya finalidad apunta a sustentar la hipótesis de que es viable y deseable incluir políticas de Alfabetización en Información en los Planes de Lectura. Se aspira a generar un modelo de Alfabetización Informacional en el contexto del Plan Nacional de Lectura de Uruguay, como una forma de contribuir al fomento de la lectura.

\section{Breve marco teórico}

A continuación se brinda un breve marco teórico que conceptualiza las grandes líneas temáticas que aborda esta investigación:

\subsection{Alfabetización Informacional}

Los escenarios globales que presenta la sociedad de la información y el conocimiento han transformado la vida de los individuos, propiciando nuevos comportamientos, formas de relacionarse, de aprender, de vivir, fuertemente condicionados al contexto que ofrecen las tecnologías de la información y la comunicación. Afirma David BAWDEN (2002) que el concepto de alfabetización ha evolucionado desde lo funcional, considerando únicamente el aprendizaje de leer y escribir, hasta incluir en el mismo, acciones como: leer, escribir, comprender, internalizar, elaborar, comunicar, evaluar, ser crítico e independiente en una sociedad en continuo cambio.

Según BENITO MORALES (2000) el concepto de alfabetización amplía su significado en la sociedad actual, ya que las habilidades de lecto-escritura se vuelven insuficientes. Se necesitan además otras capacidades para enfrentarse al inmenso caudal informativo y contar con las destrezas necesarias para recibir la información, evaluarla y ser crítico con ella.

El concepto de alfabetización está cambiando y abarca otros conceptos relacionados que surgen de una misma base pero que apuntan a objetivos diferentes. Aparecen las "alfabetizaciones en destrezas", como respuesta a las necesidades y complejidades que el medio va desarrollando y se plantean alternativas de formación que permitan un mejor relacionamiento con cada situación. Así por ejemplo, se habla de alfabetización bibliotecaria, tecnológica, electrónica, visual, informacional, académica. En este mismo marco, se confunde y relaciona directamente la alfabetización informacional con la alfabetización en el uso de las nuevas tecnologías. Sin embargo los conceptos son significativamente diferentes.

Las definiciones de lo que implica la Alfabetización Informacional son múltiples y han ido cambiando con el tiempo. Sólo a los efectos ilustrativos se transcribe la expresada en la Declaración de Alejandría (2005), que definió la Alfabetización Informacional en conexión con el aprendizaje permanente como eje en todas las facetas de la vida y "faro" de la sociedad de la información:

La alfabetización informacional se encuentra en el corazón mismo del aprendizaje a lo largo de la vida. Capacita a la gente de toda clase y condición para buscar, evaluar, 
utilizar y crear información eficazmente para conseguir sus metas personales, sociales, ocupacionales y educativas. Constituye un derecho humano básico en el mundo digital y promueve la inclusión social de todas las naciones. El aprendizaje a lo largo de la vida permite que los individuos, las comunidades y las naciones alcancen sus objetivos y aprovechen las oportunidades que surgen en un entorno global en desarrollo para beneficios compartidos. Ayuda a las personas y a sus instituciones a afrontar los retos tecnológicos, económicos y sociales, a remediar las desventajas y a mejorar el bienestar de todos. La Alfabetización Informacional incluye entre otras: a) las competencias para reconocer las necesidades de información y para localizar, evaluar, aplicar y crear información en contextos sociales y culturales; b) se extiende más allá de las meras tecnologías actuales para cubrir el aprendizaje, el pensamiento crítico y las competencias de interpretación por encima de las fronteras profesionales, potenciando a los individuos y comunidades. (Declaración de Alejandría, 2005)

Durante mucho tiempo la Alfabetización Informacional estuvo identificada con la necesidad de aprender el manejo y uso de las tecnologías de la información y por lo tanto su contenido se volcaba hacia lo informático, lo digital. Sin embargo, actualmente se ha establecido con claridad que la misma se refiere al desarrollo de habilidades y destrezas que le permiten a un individuo ser competente en su relación con la información (selección, evaluación, acceso, creación, difusión), no importando el soporte en el que se encuentre la misma, sino su contenido.

El desarrollo de las políticas de Alfabetización Informacional ha generado el diseño de distintos modelos y estándares (normas), orientados a diferentes públicos y objetivos que se vienen aplicando a nivel mundial, que aspiran a la implementación de la Alfabetización Informacional en toda su extensión. Así por ejemplo, la International Federation of Library Association (IFLA) en nombre de UNESCO lleva adelante la realización del "Information Literacy Resources Directory" (Infolit Global) ${ }^{2}$ que es una base de datos que permite difundir y consultar distintas iniciativas a nivel mundial relacionadas con Alfabetización Informacional. Es una fuente de consulta ineludible que centraliza la información de mayor actualidad y el estado del arte de la temática a nivel mundial.

Respecto al tema de Alfabetización Informacional, Uruguay está en etapa de desarrollo. Se están encaminando distintas acciones, pero aún presentan un fuerte componente que apunta esencialmente a lo tecnológico más que a los contenidos. Sólo a modo de ejemplo se menciona el Plan $\mathrm{Ceibal}^{3}$, que tiene como objetivo principal acercar la tecnología a nivel de la educación primaria, otorgando un computador personal a cada escolar a nivel nacional. Esta iniciativa está acompañada de propuestas educativas que están en plena implementación y evaluación.

\footnotetext{
${ }^{2}$ InfoLit Global.- IFLA/UNESCO. Information Literacy Resources Directory. [En línea] Disponible en url: <http:www.infolitglobal.info/>

3 Plan Ceibal.- www.ceibal.edu.uy
} 


\subsection{Lectura}

¿Cómo contribuir a la formación de individuos críticos, pensantes y autónomos que puedan ser partícipes activos de la sociedad de la información y el conocimiento?

Desde la perspectiva de este trabajo se puede afirmar que la lectura es una herramienta fundamental para dar respuesta a esta pregunta. La lectura en su expresión más amplia (formativa, recreativa, informativa), en cualquier soporte brinda a los individuos la posibilidad de analizar, interpretar, comunicar, evaluar, crear, transformar y reflexionar sobre la realidad y asimismo es un factor determinante para potenciar el crecimiento y desarrollo personal, ejercer libertades, derechos y obligaciones.

Las afirmaciones de MILLÁN (2000) sobre la importancia de la lectura son ampliamente significativas:

La llave mágica del conocimiento es la lectura. Será necesario repetirlo, porque estamos subyugados por la magnitud y las virtudes de los nuevos prodigios tecnológicos y al tiempo deberemos reaprender las potencialidades y las maravillas de algo que consideramos trivial, sólo porque lo poseemos ya y nos acompaña desde hace muchísimo tiempo". ... “LA LECTURA ES LA LLAVE DEL CONOCIMIENTO EN LA SOCIEDAD DE LA INFORMACIÓN.” ..."El desarrollo humano no avanza en zigzag ni a salto, sino que normalmente construye sobre lo anterior. La lucha por comprender y utilizar las nuevas tecnologías digitales exige muchas cosas nuevas, si: pero presupone las antiguas. Y la más importante de ellas es la lectura. (MILlÁN, 2000)

La lectura debe ser visualizada desde sus múltiples dimensiones y no restringirla solamente al ámbito educativo y/o recreativo. No siempre el individuo es consciente del lugar que ella ocupa en su vida. Es habitual que se escuche la afirmación "yo no leo" o "no soy lector". Estas afirmaciones generalmente se hacen considerando el referente que la sociedad tiene respecto a lo que significa ser lector. Se asocia el gusto por la lectura específicamente a un tipo de lectura, la recreativa (novela, ficción, poesía, etc.) y no se tiene en cuenta que continuamente se hace uso de la herramienta lectura.

Durante muchos siglos leer y escribir fue privilegio de unos pocos. La lectura entendida como una forma de libertad del individuo es muy reciente y tiene relación directa con el derecho que tiene cada persona de desarrollar sus propias capacidades intelectuales, a aprender y crecer como individuo. Que la lectura se vuelva popular resultaba peligroso para algunos sectores más poderosos de la sociedad, y es así que surgen lo que CHARTIER Y HEBRARD (2000) llaman discursos sobre la lectura. O sea, se elaboran fundamentos teóricos a favor y en contra de que la lectura esté al alcance de todos y en especial se enfatiza en qué textos son los se puede o no leer.

A fines del siglo XIX y principios del siglo XX, se difunde e introduce con fuerza el modelo norteamericano en casi todos los países, que promueve la lectura pública moderna basado en la libertad de elección y acceso a lo que se quiere leer. Este modelo produjo un cambio significativo en la profesión de bibliotecario que deja de ser la de custodia de los materiales de la biblioteca para convertirse en el mediador entre el lector y la lectura: un verdadero promotor de lectura. Se comienza a hablar de la lectura de actualidad y de información. Ésta última es la lectura de 
consulta, que no necesariamente es para el estudio o el entretenimiento (CHARTIER Y HEBRARD, 2000).

A mediados del siglo XX la lectura trasciende la escuela y pasa a ser el objeto de estudio de distintas disciplinas, que analizan el proceso de la misma desde distintas ópticas, entre ellas la psicología y la sociología. En este nuevo contexto el modelo moderno de la lectura, la libertad para leer y la lectura como medio para lograr el crecimiento personal del individuo y no sólo para aplicarla en la escuela, impregna los objetivos de quienes aspiran a que la gente lea.

Así es que adquieren gran relevancia las bibliotecas públicas, ya que brindan la posibilidad de que todos los ciudadanos tengan acceso a la lectura y que en ese medio puedan ejercer sus libertades y derechos. Entonces, la lectura se vuelve un medio para garantizar la igualdad social, la democratización de la información y la construcción del conocimiento. Leer para informarse, para formarse, por placer. El lector debe tener la libertad de elegir que lee y como lo lee.

Expresa Daniel CASSANY:

Todavía hoy muchas personas creen que leer consiste en oralizar la grafía, en devolver la voz a la letra callada. Se trata de una concepción medieval, que ya hace mucho que la ciencia desechó. Es una visión mecánica que pone el acento en la capacidad de descodificar la prosa de modo literal. Sin duda deja en un segundo plano a la comprensión- que es lo importante". Y continúa expresando "Más moderna y científica es la visión de que leer es comprender. Para comprender es necesario desarrollar varias destrezas mentales o procesos cognitivos: anticipar lo que dirá un escrito, aportar nuestros conocimientos previos, hacer hipótesis y verificarlas, elaborar inferencias para comprender que sólo se sugiere, construir un significado, etc. Llamamos alfabetización funcional a este conjunto de destrezas, a la capacidad de comprender el significado de un texto. Y denominamos analfabeto funcional a quien no puede comprender la prosa, aunque pueda oralizarla en voz alta. (CASSANY, 2006)

Abordar el tema de la lectura es especialmente complejo y no es posible hacerlo desde una visión única, estática o a través de una sola disciplina. Las concepciones sobre lectura son múltiples, cambiantes y se manifiestan desde variados puntos vista y se transforman y responden a los distintos períodos históricos, tendencias y evolución de la sociedad. En este sentido BAVOSI (2006) expresa que el tema de la lectura ha sido y es abordado desde múltiples visiones, educativas, pedagógicas, psicológicas, sociales, lingüísticas, pero a pesar de ello, sigue siendo un problema.

La lectura es fundamental en el mundo actual en el que priman las nuevas tecnologías de la información y la comunicación. Ellas generan una forma de lectura en la que no sólo es necesario comprender, sino saber seleccionar y encontrar lo que se busca, que no siempre resulta fácil. Este es un punto clave de vínculo entre la lectura y la Alfabetización Informacional. Las nuevas modalidades de lectura exigen mayor atención en la selección y evaluación de lo que se lee ya que el volumen de información que se maneja es incontrolable. Internet, sitios web, chats, correos electrónicos, mensajes por celular, se presentan con nuevas estructuras de formulación, con menos formalidades y accesibles a quien tenga la posibilidad de contar con conexión. CASSANY (2006) 


\subsection{Promoción de la lectura: políticas y planes}

En los años ochenta del siglo pasado, se apunta a posicionar a la lectura como herramienta de integración de los individuos a la sociedad. Se llevan adelante importantes estudios que brindan datos objetivos que confirman que las personas no leen, o leen poco, que algunos leen pero no comprenden y que hay un número elevado de analfabetos, pero también de analfabetos por desuso, aquellos que aprendieron la técnica de la escritura y la lectura, pero no la practican habitualmente. El problema del analfabetismo se convierte en el centro de atención y la preocupación por acercar la lectura a las personas, en especial desde la infancia, se vuelve un objetivo generalizado de la educación y de otras áreas de la sociedad civil, pero también paulatinamente de los gobiernos. Comienza un período en el que se toma conciencia de la situación generada en torno a la lectura y cada vez con más fuerza, sobre todo en el ámbito de la educación y del mercado editorial se manejan distintas estrategias que colocan a la lectura en su centro de atención.

Se promueven distintas iniciativas que van desde campañas de lectura puntuales, pasando por acciones de fomento y promoción, hasta la formulación de políticas públicas de lectura en las que los gobiernos comprometen sus intereses, iniciativas y esfuerzos en función de beneficiar a la sociedad en su conjunto con diferentes acciones en favor del fomento lector.

Generalmente cuando se piensa en promoción de la lectura se asocia con estrategias de animación que inducen a la lectura especialmente de libros y por placer. Pero este es sólo un aspecto de los muchos que pueden contribuir a impulsar al individuo hacia la lectura y en este sentido se afirma que las acciones de Alfabetización Informacional son una forma más de promoción de la lectura. Esta afirmación se hace más evidente cuando la promoción de la lectura se enfoca como una política pública y pretende alcanzar objetivos que beneficien a toda la comunidad.

¿Y qué implica decir que existe una política pública de promoción de la lectura? Implica el establecimiento de objetivos claros y orientaciones estratégicas que se formulan con la finalidad de planificar, articular e implementar acciones en favor de la lectura. Cuando el estado decide abordar un tema desde la dimensión de una política pública, significa que ese tema es de interés social y su producto se manifiesta como una construcción colectiva. Una política pública de lectura debe basarse en que la misma es responsabilidad de la sociedad en su conjunto, valorizando su importancia para el ejercicio democrático de acceso al conocimiento, considerando la diversidad cultural y los distintos actores que en ella deben estar involucrados. La política pública de lectura debe dirigirse a la familia, la escuela, la biblioteca, espacios de la comunidad, además de la creación, producción y circulación de los materiales de lectura.

De acuerdo a estudios realizados por la CERLALC (1999), los antecedentes sobre políticas públicas de lectura en América Latina se remontan a los ańos 1980, en donde surgen asociaciones profesionales, creación de salas de lectura infantil en distintas bibliotecas, se impulsa a los autores, escritores e ilustradores en especial hacia la producción infantil y juvenil, se produce un gran desarrollo de la industria gráfica y editorial y se inician los movimientos de transformación de la Escuela. Acompañando estos cambios se trabaja en la creación de bibliotecas públicas y populares como ámbitos naturales de promoción de la lectura. 
A nivel de Iberoamérica se destacan las políticas de promoción de la lectura que llevan adelante, Brasil, Chile, España y México a través de sus Planes Nacionales de Lectura. El Plan Nacional de Lectura de Brasil, hace mención expresa a la necesidad de implementar políticas de Alfabetización Informacional.

\subsubsection{Plan Nacional de Lectura de Uruguay}

Uruguay no ha permanecido ajeno a las iniciativas mundiales y regionales de promover acciones a favor de la lectura. Las mismas surgen alrededor de los ańos 70 del siglo XX, como respuesta a una serie de variables que fundamentan la preocupación porque los individuos no leen.

Recién a principios del año 2005 se puede hablar de la existencia de una política pública nacional de lectura, con la creación y puesta en funcionamiento del Plan Nacional de Lectura promovido por la Dirección de Educación del Ministerio de Educación y Cultura. Este es un hito en la historia de la promoción de la lectura en Uruguay, pues por primera vez se visualiza la posibilidad de encarar el tema con compromiso del Estado.

La propuesta rescata la importancia de las políticas públicas de lectura como la herramienta en la que se debe reconocer el papel fundamental que en ese marco desempeñan las instituciones del Estado, el sector privado, los organismos internacionales y las organizaciones de la sociedad civil.

El Plan Nacional de Lectura (2005) está pensado como política pública a largo plazo y de carácter permanente que debe surgir del debate entre distintos actores de todos los niveles, articulando esfuerzos, experiencias y recursos a nivel público y privado.

Asimismo, la política pública de lectura que se pretende implementar en Uruguay apunta especialmente a favorecer la inclusión social y la democratización del conocimiento. La lectura como herramienta de inclusión social implica reconocer que es derecho de todas las personas y que a través de ella se desarrolla el potencial humano; que la lectura permite alcanzar mejores niveles educativos y por lo tanto es fundamental en la construcción de la sociedad democrática. Los lineamientos generales que la Dirección de Educación dio al Plan se basaron en dos ejes principales y transversales: articulación y divulgación.

A partir de estos ejes el Plan procura constituirse en una guía de actividades concretas consideradas prioritarias y realizables y cada año se desarrolla un plan de trabajo concreto en función de los mismos. Los principales ejes de acción son: la formación de recursos humanos; la investigación en el área; la lectura y las tecnologías de la información y la comunicación; las bibliotecas escolares; el fortalecimiento de acervos y la difusión y sensibilización.

La experiencia desarrollada por la EUBCA en el marco del Plan Nacional de Lectura, responde a los conceptos vertidos anteriormente y refleja una postura de la Bibliotecología en cuanto al trabajo con la comunidad desde la integración multidisciplinar. La misma ha sido encarada como una experiencia piloto, evaluable que aspira a diseńar un modelo que contenga pautas que orienten la implementación de acciones de promoción de la lectura y Alfabetización Informacional, tomando como centro de referencia la biblioteca. 
La experiencia se viene desarrollando bajo la responsabilidad de un equipo interdisciplinar en el que participan docentes de la EUBCA que pertenecen a distintas áreas temáticas de la bibliotecología tales como: lectura, literatura infantil, recursos de información y referencia, formación de usuarios, procesos técnicos, administración, relacionamiento con la comunidad. Al equipo docente se han integrado estudiantes de la Licenciatura en Bibliotecología que están trabajando a modo de práctica curricular en la que se conjugan aspectos teórico-prácticos de su formación académica.

Se aspira a diseñar un "modelo base" de promoción de la lectura a nivel nacional, que pueda ser aplicado en el marco de una política pública de lectura que incorpore políticas de Alfabetización Informacional. Considerando la diversidad de la sociedad en su conjunto no se cree conveniente pensar en un modelo único, sino en pautas generales que acompańen los procesos particulares de cada comunidad.

\section{La Promoción de la Lectura y la Alfabetización Informacional}

La bibliografía internacional que relaciona la promoción de la lectura y la Alfabetización Informacional es escasa y reciente, ya que es un tema en construcción y sobre el cual recién empiezan a generarse los primeros conocimientos. Sin embargo, es de destacar la investigación realizada por la Dra. Aurora Cuevas Cervero, sobre lectura Alfabetización Informacional y biblioteca escolar, ${ }^{4}$ que resulta una valiosa contribución al desarrollo del tema.

La lectura proporciona al individuo las condiciones para integrarse activamente a la sociedad. El individuo que comprende lo que lee puede generar conocimiento. En la sociedad actual, el acceso a la información se produce a través de la lectura ya sea de textos o imágenes, en soporte manual o electrónico. La presencia de las nuevas tecnologías de la información y la comunicación han revalorizado la importancia de la lectura.

La lectura posibilita el aprendizaje a lo largo de toda la vida, favorece la libertad intelectual, el pensamiento crítico y estimula el desarrollo personal. En el sentido más amplio del término un individuo alfabetizado no sólo estará apto para leer y escribir, sino que estará en condiciones de analizar, comprender, interpretar, crear el mundo que lo rodea y ser parte activa del mismo. Siguiendo a HERNANDEZ SÁNCHEZ (2001), la práctica de la lectura transforma la información en conocimiento.

Por su parte la Alfabetización Informacional promueve la formación de individuos críticos, pensantes, capaces de enfrentarse a los retos y cambios constantes de la sociedad actual. Apunta a que el individuo reconozca sus necesidades de información, y aprenda a usar, seleccionar y evaluar esa información en forma crítica. La alfabetización informacional es la base del aprendizaje a lo largo de toda la vida y permite al individuo crecer a nivel personal y contribuir al desarrollo social. Estar alfabetizado en información va mucho más allá de saber usar las tecnologías de la información y la comunicación. Adquirir competencias específicas para relacionarse con la información debe ser un

\footnotetext{
${ }^{4}$ CUEVAS CERVERO, Aurora (2005). La promoción de la lectura como modelo de alfabetización en información en bibliotecas escolares. Madrid: Trea. Tesis Doctoral. Universidad Carlos III de Madrid.
} 
objetivo tanto a nivel de la educación formal como informal y para ello se necesita también competencia lectora.

La biblioteca es el ámbito natural para la implementación de políticas de promoción de la lectura y de Alfabetización Informacional. A través de ella se puede sustentar el aprendizaje a lo largo de toda la vida; fomentar la inclusión social, la integración multicultural y la democratización de la información y por lo tanto del conocimiento. La biblioteca debe ocupar un lugar de preeminencia en el contexto de las políticas culturales, educativas e informativas de un país. Dice MERLO VEGA (2006) que las bibliotecas públicas y la promoción de la lectura son dos realidades indisolubles.

$\mathrm{Al}$ mismo tiempo, las bibliotecas son espacios apropiados para llevar adelante políticas de Alfabetización Informacional y en los hechos durante años han impulsado este tipo de actividades, bajo la denominación de formación de usuarios en el uso de la información. La formación de usuarios podría considerarse el antecedente más directo de la Alfabetización Informacional.

Por su parte, del Seminario sobre "Bibliotecas, aprendizaje y ciudadanía: la alfabetización informacional”, realizado en Toledo en 2006 del cual surge la Declaración de Toledo sobre Alfabetización Informacional (2006), realiza una serie de afirmaciones que posicionan a la biblioteca en un rol fundamental en la educación, la alfabetización informacional y la promoción de la lectura.

\section{Consideraciones finales}

Sin dudas, se puede afirmar la viabilidad de la implementación de políticas de Alfabetización Informacional en el marco del Plan Nacional de Lectura, que tome como base la infraestructura de las bibliotecas, podría favorecer ampliamente las acciones de promoción de la lectura y la articulación y coordinación de políticas informativas, educativas y sociales a nivel del país.

Generalmente, los Planes de Lectura se estructuran teniendo en cuenta algunos principios básicos que se reiteran, con mayor o menor énfasis, en todos ellos. Esos aspectos son: el objetivo principal de favorecer la formación de individuos pensantes, críticos, y aptos para el aprendizaje a lo largo de toda la vida, permitiendo la democratización del acceso a la información en función de la generación de conocimiento y la inclusión social; la capacitación de promotores de lectura, la existencia de bibliotecas, la integración del mercado editorial, y líneas de investigaciones sobre el tema. Pero, las políticas de Alfabetización Informacional no aparecen relacionadas, ni mencionadas en los Planes Nacionales de Lectura.

Se estima que los argumentos expuestos precedentemente expresan fuertes fundamentos que podrían dar base a una propuesta que contemple el vínculo entre la promoción de la lectura y la Alfabetización Informacional, tomando como punto de partida el espacio de la biblioteca en el marco del Plan Nacional de Lectura, y que permitiera generar un modelo de ALFIN propio para Uruguay. Demás está decir que el desarrollo teórico expuesto es sólo el principio para analizar la viabilidad de una propuesta y formular una visión global de la promoción de la lectura. La investigación en curso aspira a generar el conocimiento y los insumos necesarios para sustentar esta hipótesis de trabajo. 


\section{Referencias biliográficas}

BAVOSI, Ana María. Leer o no leer: ¿a quién le importa? (2006). Revista de la Educación del Pueblo. Montevideo. 2a. Ép. No. 101. p. 6-8.

BADWEN, David (2002) Revisión de conceptos de alfabetización informacional y digital. Anales de Documentación. No. 5, p. 361-408.

BENITO MORALES, Félix (2000). Nuevas necesidades, nuevas habilidades.

Fundamentos de la alfabetización informacional. En: José A. Gómez Hernández, coord. Estrategias y modelos para enseñar a usar la información. Murcia: KR.

BROVETTO, Jorge (1994). Formar para lo desconocido. Montevideo: Universidad de la República.

CASSANY, Daniel (2006). Tras las líneas: sobre la lectura contemporánea.- Barcelona: Anagrama. $294 \mathrm{p}$.

CERLALC (1999). Las políticas nacionales de lectura.- 2a. ed. Bogotá: Cerlalc.

CHARTIER, Anne-Marie; HÉBRARD, Jean (2000). La lectura de un siglo a otro: discursos sobre la lectura (1980-2000). Barcelona: Gedisa, 204 p.

MANIFIESTO DE ALEJANDRÍA SOBRE BIBLIOTECAS: la Sociedad de la Información en acción. IFLA. (2005). Disponible en: http://www.ifla.org/III/wsis/AlexandriaManifestoes.html

MARZAL, Miguel Ángel (2004). Investigación para la formación de bibliotecarios y documentarias en alfabetización informacional. En: Universidad de Murcia, ANABAD, Consejería de Educación y Cultura de la Región de Murcia, Dirección General del Libro, Archivos y Bibliotecas del Ministerio de Cultura (eds). Foro Biblioteca y Sociedad. Experiencias de Innovación y Mejora. Murcia: 14-16 de octubre de 2004. CD-ROM.

MATTELART, Armand (2004). La sociedad global de la información es un mito.

Disponible en: http://voltairenet.org/article121544.html

MERLO VEGA, Antonio (2006). La Biblioteca pública como promotora de la lectura: planes de lectura y experiencias de fomento lector en Espańa.

Disponible en: http://www.congresobibliotecaspublicasdechile.cl/ponencias/MerloJoseAntonio. pdf

MILLAN, José Antonio (2000). La lectura y la sociedad del conocimiento. Disponible en: http://jamillan.com/lecsoco.htm

MINISTERIO DE EDUCACIÓN Y CULTURA. URUGUAY. (2005). Plan Nacional de Lectura. Montevideo. Disponible en: http://www.mec.gub.uy/ 